Journal of Applied ANALysis

Vol. 7, No. 1 (2001), pp. 43-59

\title{
GENERALIZED SOLUTIONS OF A PERIODIC GOURSAT PROBLEM
}

\author{
V. VALMORIN \\ Received March 3, 2000 and, in revised form, November 28, 2000
}

\begin{abstract}
Under reasonable assumptions on the data $u, v$ and the function $f$, we show that the nonlinear periodic Goursat problem

$$
\begin{aligned}
& \frac{\partial^{2} u}{\partial x \partial y}(x, y)=f(x, y, u(x, y)) ; \\
& u(x, 0)=v(x) ; \quad u(0, y)=w(y)
\end{aligned}
$$

which cannot be posed in the general theory of distributions, may be studied and solved in a differential algebra of periodic new generalized functions on $\mathbb{R}^{2}$. This algebra contains, in a canonical way, the space of periodic distributions on $\mathbb{R}^{2}$ as a linear subspace. When the data are Dirac measures, a particular study is done, showing the nature of the singularities involved in this case.
\end{abstract}

1991 Mathematics Subject Classification. 46F10, 42B05, 35D99.

Key words and phrases. Periodic distributions, periodic generalized functions, differential problem, nonlinearity, strong singulatities.

ISSN 1425-6908（C) Heldermann Verlag. 


\section{Introduction}

We consider in $\mathbb{R}^{2}$ the following so-called Goursat Problem $[7,8,13,15]$ :

$$
\begin{cases}\frac{\partial^{2} u}{\partial x \partial y}(x, y) & =f(x, y, u(x, y)) \\ u(x, 0) & =v(x) \\ u(0, y) & =w(y) .\end{cases}
$$

In this system $f$ is a complex valued function on $\mathbb{R}^{2} \times \mathbb{C}$, smooth in the four underlying real variables. $v$ and $w$ are intended to belong to some space of $2 \pi$-periodic functions on $\mathbb{R}$, where functions are considered in a generalized sense. It is immediately seen that the condition $v(0)=w(0)$ must hold because of the two last equalities of the system. However, if $v$ and $w$ are distributions, it is not allways true that this condition makes sense. In the same way the consideration of $u_{\mid\{y=0\}}$ or $u_{\mid\{x=0\}}$ leads to the same type of difficulty. We also note that difficulties may arise from the possible nonlinearities carried by the function $f$.

Therefore, the global investigation of such a Goursat problem needs a more general framework that the one of distributions theory. So we have to study a problem which may be solved by considering the recent theory of new generalized functions. See references in this paper an particularly $[9,10]$ for a comprehensive account of this topic. Since in our situation, we have to take periodicity in account, we shall work in an algebra of periodic generalized functions. In a previous paper [14], we have constructed a differential algebra $\mathcal{G}_{n} ; n=1,2, \ldots$ of $2 \pi$-periodic new generalized functions on $\mathbb{R}^{n}$ in which the space of $2 \pi$-periodic distributions on $\mathbb{R}^{n}$ is canonically embedded as a linear subspace. This gives us the good setting to deal with our Goursat problem. We take the data $v$ and $w$ in $\mathcal{G}_{1}$, and seek a solution $u$ in $\mathcal{G}_{2}$. Using the above-mentioned canonical embedding, we may consider the case where $v$ and $w$ are distributions and especially Dirac measures.

In the first section of the present paper, we give without proofs the necessary results needed on the differential algebras $\mathcal{G}_{n}$. More results and proofs can be found in [14]. The second section is devoted to our main purpose; the study of the generalized Goursat problem. The main results on existence and uniqueness of the solution $u$ are stated in the second section, their proofs being given at the end.

\section{The algebra $\mathcal{G}_{n}$}

This section summarizes the results obtained in [14] which are necessary for our purpose. More details and proofs can be found in this paper. 
Construction of $\mathcal{G}_{n}$. We set $\mathcal{I}=[-\pi, \pi]$. If $n \in$ is a positive integer, $\mathcal{E}_{2 \pi}^{(n)}$ denotes the algebra of smooth complex-valued and $2 \pi$-periodic functions on $\mathbb{R}^{n}$ equipped with the topology of uniform convergence of functions and their derivatives. If $u \in \mathcal{E}_{2 \pi}^{(n)}$ and $\alpha \in \mathbb{N}^{n}$ we set $\partial^{\alpha} u=\left(\partial / \partial x_{1}\right)^{\alpha_{1}} \ldots$ $\left(\partial / \partial x_{n}\right)^{\alpha_{n}} u,\|u\|_{\infty}=\sup _{x \in \mathcal{I}^{n}}|u(x)|$. The space $\mathcal{D}_{2 \pi}^{\prime(n)}$ of $2 \pi$-periodic distributions on $\mathbb{R}^{n}$ is the topological dual of $\mathcal{E}_{2 \pi}^{(n)}$. If $p \in \mathbb{Z}^{n}, x \in \mathbb{R}^{n}$ and $T \in \mathcal{D}_{2 \pi}^{\prime(n)}$, the Fourier coefficient of index $p$ of $T$ is denoted by $\widehat{T}(p)$ or $\widehat{T}_{p}$ and $e^{i p x}$ by $e_{p}(x)$ where $p x=p_{1} x_{1}+\ldots+p_{n} x_{n}$. The periodic Dirac measure $\sum_{p \in \mathbb{Z}^{n}} e_{p}$ will be denoted by $\delta_{n}$ or $\delta$ if $n=1$.

Let $m \in \mathbb{N}, I_{m}=\{p \in \mathbb{Z}:|p| \leq m\}, n \in \mathbb{N}^{*}$ and $\chi \in \mathcal{E}_{2 \pi}^{(1)}$. We define the following subsets:

$$
\begin{aligned}
\mathcal{A}_{m}^{(1)} & =\left\{\varphi \in \mathcal{E}_{2 \pi}^{(1)}: \widehat{\varphi}(p)=1 \text { if } p \in I_{m}\right\} ; \\
\mathcal{A}_{m}^{(n)} & =\left\{\varphi \in \mathcal{E}_{2 \pi}^{(n)}: \exists \psi \in \mathcal{A}_{m}^{(1)}, \varphi=\psi^{\otimes n}\right\} ; \\
\mathcal{A}^{(n)} & =\bigcup_{m \in \mathbb{N}} \mathcal{A}_{m}^{(n)} ; \\
\mathcal{A}_{m}^{(1)}(\chi) & =\left\{\varphi \in \mathcal{E}_{2 \pi}^{(1)}: \widehat{\varphi}(p)=1 \text { if } p \in I_{m},|\widehat{\varphi}(p)| \leq|\widehat{\chi}(p)| \text { if } p \notin I_{m}\right\} ; \\
\mathcal{A}_{m}^{(n)}(\chi) & =\left\{\varphi \in \mathcal{E}_{2 \pi}^{(n)}: \exists \psi \in \mathcal{A}_{m}^{(1)}(\chi), \varphi=\psi^{\otimes n}\right\} .
\end{aligned}
$$

We note that $\mathcal{A}_{m}^{(n)}=\cup_{\chi \in \mathcal{E}_{2 \pi}^{(1)}} \mathcal{A}_{m}^{(n)}(\chi)$. Let $\psi \in \mathcal{E}_{2 \pi}^{(1)}$ and $\varphi=\psi^{\otimes n}$. We have $\widehat{\varphi}(p)=\widehat{\psi}\left(p_{1}\right) \ldots \widehat{\psi}\left(p_{n}\right)=\widehat{\psi}^{\otimes n}(p)$ so that if $\varphi \in \mathcal{A}_{m}^{(n)}$ then $\widehat{\varphi}(p)=1$ for $p \in I_{m}$; if $\varphi \in \mathcal{A}_{m}^{(n)}(\chi)$ then $\widehat{\varphi}(p)=1$ for $p \in I_{m}$ and $|\widehat{\varphi}(p)| \leq\left|\widehat{\chi}^{\otimes n}(p)\right|$ for $p \notin I_{m}$. Let $\rho_{m}=\sum_{p \in I_{m}} e_{p}$. Clearly we have that $\rho_{m} \in \mathcal{A}_{m}^{(1)}$ and then $\rho_{m}^{\otimes n} \in$ $\mathcal{A}_{m}^{(n)}$. We note that $\rho_{m}(t)=[\sin (m+1 / 2) t] / \sin (t / 2)$ and $\lim _{m \rightarrow \infty} \rho_{m}^{\otimes n}=\delta_{n}$ in $\mathcal{D}_{2 \pi}^{\prime(n)}$.

Let $\mathcal{X}_{n}$ denote the algebra of $\mathcal{E}_{2 \pi}^{(n)}-$ valued maps on $\mathcal{A}^{(n)}$. We define

$$
\begin{aligned}
& \mathcal{X}^{(n)}=\left\{\begin{array}{l}
u \in \mathcal{X}_{n}: \forall \chi \in \mathcal{E}_{2 \pi}^{(1)}, \forall \alpha \in \mathbb{N}^{n}, \exists r \in \mathbb{R}, \exists N \in \mathbb{N}, \exists c>0: \\
\forall m \geq N, \varphi \in \mathcal{A}_{m}^{(n)}(\chi) \Longrightarrow\left\|\partial^{\alpha}(u(\varphi))\right\|_{\infty} \leq c(m+1)^{r}
\end{array}\right\} ; \\
& \mathcal{N}^{(n)}=\left\{\begin{array}{l}
u \in \mathcal{X}_{n}: \forall \chi \in \mathcal{E}_{2 \pi}^{(1)}, \forall \alpha \in \mathbb{N}^{n}, \forall q>0, \exists N \in \mathbb{N}, \exists c>0: \\
\forall m \geq N, \varphi \in \mathcal{A}_{m}^{(n)}(\chi) \Longrightarrow\left\|\partial^{\alpha}(u(\varphi))\right\|_{\infty} \leq c(m+1)^{-q}
\end{array}\right\} .
\end{aligned}
$$

Proposition 1.1. $\mathcal{X}^{(n)}$ is a subalgebra of $\mathcal{X}_{n}$ and $\mathcal{N}^{(n)}$ a subalgebra of $\mathcal{X}^{(n)}$.

Now, the algebra $\mathcal{G}_{n}$ is defined as follows: 
Definition 1.1. The algebra $\mathcal{G}_{n}$ of periodic generalized functions is the factor algebra $\mathcal{X}^{(n)} / \mathcal{N}^{(n)}$.

If $u \in \mathcal{X}_{n}$ and $x \in \mathbb{R}^{n}$ we set $u=\left(u_{\varphi}\right)_{\varphi \in \mathcal{A}^{(n)}}$ or $u=\left(u_{\varphi}\right)_{\varphi},[u(\varphi)](x)=$ $u(\varphi, x)$ and for any $\alpha \in \mathbb{N}^{n}$ we define $\partial^{\alpha} u$ as $\left(\partial^{\alpha} u_{\varphi}\right)_{\varphi}$. Clearly it holds $\partial^{\alpha}\left(\mathcal{X}^{(n)}\right) \subset \mathcal{X}^{(n)}$. So if $U \in \mathcal{G}_{n}$ and $u$ is a representative of $U$, writing $\operatorname{cl}(u)$ to denote the class of $u$, we can consider $\operatorname{cl}\left(\partial^{\alpha} u\right)$. Because $\partial^{\alpha}\left(\mathcal{N}^{(n)}\right) \subset \mathcal{N}^{(n)}$, $\operatorname{cl}\left(\partial^{\alpha} u\right)$ is independent of the chosen representative, then if we define $\partial^{\alpha} U$ as $\operatorname{cl}\left(\partial^{\alpha} u\right), \mathcal{G}_{n}$ becomes a differential algebra which is obviously associative and commutative with $\operatorname{cl}\left(1_{\varphi}\right)_{\varphi}$ as unit element where $1_{\varphi}=1$ for any $\varphi$ in $\mathcal{A}^{(n)}$.

Basic properties of $\mathcal{G}_{n}$. If $\mathcal{I}=[-\pi, \pi]$, we recall that the convolution of two elements $f$ and $g$ in $\mathcal{E}_{2 \pi}^{(n)}$ is defined by

$$
(f * g)(x)=\left(\frac{1}{2 \pi}\right)^{n} \int_{\mathcal{I}^{n}} f(x-y) g(y) d y=\left(\frac{1}{2 \pi}\right)^{n} \int_{\mathcal{I}^{n}} f(y) g(x-y) d y .
$$

For $T \in \mathcal{D}_{2 \pi}^{\prime(n)}$ and $\varphi \in \mathcal{E}_{2 \pi}^{(n)}$, the convolution $T * \varphi=\sum \widehat{T}(p) \widehat{\varphi}(p) e_{p}$ belongs to $\mathcal{E}_{2 \pi}^{(n)}$, and $\langle T, \varphi\rangle=\sum \widehat{T}(p) \widehat{\varphi}(-p)$. The canonical linear embedding of $\mathcal{D}_{2 \pi}^{\prime(n)}$ in $\mathcal{G}_{n}$ is given in the following

Proposition 1.2. If $T \in \mathcal{D}_{2 \pi}^{\prime(n)}$, then $(T * \varphi)_{\varphi} \in \mathcal{X}^{(n)}$. Moreover, the map $\mathbf{i}: \mathcal{D}_{2 \pi}^{\prime(n)} \longrightarrow \mathcal{X}^{(n)}$ such that $\mathbf{i}(T)=(T * \varphi)_{\varphi}$ is linear, one-to-one and satisfies $\mathbf{i}^{-1}\left(\mathcal{N}^{(n)}\right)=\{0\}$.

The linear map $\mathbf{j}: \mathcal{D}_{2 \pi}^{\prime(n)} \longrightarrow \mathcal{G}_{n}$ such that $\mathbf{i}(T)=\operatorname{cl}(\mathbf{i}(T))$ is one-to-one and the relation $\mathbf{j}\left(\partial^{\alpha} T\right)=\partial^{\alpha}(\mathbf{j}(T))$ holds true for any $\alpha \in \mathbb{N}^{n}$.

For $f \in \mathcal{E}_{2 \pi}^{(n)}$, let $(f)_{\varphi}$ denote the sequence taking the constant value $f$. Clearly $(f)_{\varphi} \in \mathcal{X}^{(n)}$, therefore we can consider $\operatorname{cl}\left((f)_{\varphi}\right)$ in $\mathcal{G}_{n}$ and we have:

Proposition 1.3. If $f \in \mathcal{E}_{2 \pi}^{(n)}$ then $(f * \varphi-f)_{\varphi} \in \mathcal{N}^{(n)}$. Consequently the map $\sigma: \mathcal{E}_{2 \pi}^{(n)} \longrightarrow \mathcal{G}_{n}$ defined by $\sigma(f)=\operatorname{cl}\left((f)_{\varphi}\right)$ is a one-to-one morphism of differential algebras and $\mathbf{j}_{\mid \mathcal{E}_{2 \pi}^{(n)}}=\sigma$.

Association. Beside the natural equality in $U \in \mathcal{G}_{n}$, there is a weak equality based on distributions defined as follows: 
Definition 1.2. An element $U \in \mathcal{G}_{n}$ is said to admit $T \in \mathcal{D}_{2 \pi}^{\prime(n)}$ as an associated distribution if $U$ has a representative $u$ such that

$$
\forall f \in \mathcal{E}_{2 \pi}^{(n)}, \lim _{m \rightarrow \infty}\left(\frac{1}{2 \pi}\right)^{n} \int_{\mathcal{I}^{n}}\left[u\left(\rho_{m}^{\otimes n}\right)\right](x) f(x) d x=\langle T, f\rangle .
$$

This relation called association will be denoted by $U \approx T$.

This definition is independant of the chosen representative and it is easy to see that every distribution is associated with itself that is $\bar{i}(T) \approx T$ for $T \in \mathcal{D}_{2 \pi}^{\prime(n)}$.

Definition 1.3. Two elements $U$ and $V$ in $\mathcal{G}_{n}$ are said to be associated or weakly equal if $U-V$ is associated with 0 , in this case we write $U \approx V$.

Obviously association is an equivalence relation which is compatible with addition and differentiation, but it may be seen that this is not the case for multiplication. An important exception, however, is formulated in the next proposition.

Proposition 1.4. If $f \in \mathcal{E}_{2 \pi}^{(n)}$ and $T \in \mathcal{D}_{2 \pi}^{\prime(n)}$, then $\mathbf{j}(f) \mathbf{j}(T) \approx f T$.

Restriction to subspaces. Let $k, n$ be integers such that $1 \leq k<n$ and $U \in \mathcal{G}_{n}$. We denote by $u$ a representative of $U$ and by $\mathcal{F}$ the subspace $\left\{x \in \mathbb{R}^{n} / x_{k+1}, \ldots, x_{n}=0\right\}$ of $\mathbb{R}^{n}$ that we shall identify with $\mathbb{R}^{k}$. We define the restriction of $u$ to $\mathcal{F}$ as the element of $\mathcal{X}^{(k)}$, denoted by $u_{\mid \mathcal{F}}$, such that for any $\chi \in \mathcal{A}^{(n)}$

$$
\left(u_{\mid F}\right)\left(\chi^{\otimes k}\right)=\left[u\left(\chi^{\otimes n}\right)\right]_{\mid \mathcal{F}} .
$$

If $v \in \mathcal{X}^{(n)}$ and $u-v \in \mathcal{N}^{(n)}$ then $u_{\mid \mathcal{F}}-u_{\mid \mathcal{F}}=(u-v)_{\mid \mathcal{F}} \in \mathcal{N}^{(k)}$ so we have the following definition.

Definition 1.4. If $U \in \mathcal{G}_{n}$ and $u$ is a representative of $U$, then the restriction of $U$ to the subspace $\mathcal{F}$, denoted by $U_{\mid \mathcal{F}}$, is the element of $\mathcal{G}_{k}$ defined by $U_{\mid \mathcal{F}}=\operatorname{cl}\left(u_{\mid \mathcal{F}}\right)$.

If no confusion can arise, for any strictly positive integer $l$ we denote the embedding of $\mathcal{D}_{2 \pi}^{\prime(l)}$ in $\mathcal{G}_{l}$ by the letter $\mathbf{j}$. We have the following result of coherence:

\section{Theorem 1.5.}

(a) If $f$ is an element of $\mathcal{E}_{2 \pi}^{(n)}$, then $\mathbf{j}(f)_{\mid \mathcal{F}}=\mathbf{j}\left(f_{\mid \mathcal{F}}\right)$. 
(b) If $T \in \mathcal{D}_{2 \pi}^{\prime(n)}$ is a continuous function with continuous partial derivatives of order $\leq l$ with respect to the last $n-k$ variables where $l>$ $(n-k) / 2$, then $\mathbf{j}(T)_{\mid \mathcal{F}} \approx \mathbf{j}\left(T_{\mid \mathcal{F}}\right)$.

(c) If $T \in \mathcal{D}_{2 \pi}^{\prime(n)}$ is continuous with $\sum|\widehat{T}(p)|<\infty$, then $\mathbf{j}(T)_{\mid \mathcal{F}} \approx \mathbf{j}\left(T_{\mid \mathcal{F}}\right)$.

Generalized complex numbers and pointvalues. Let $\mathcal{C}$ denote the algebra of complex-valued maps $\lambda$ defined on $\mathcal{A}^{(1)}$ such that

$$
\forall \chi \in \mathcal{E}_{2 \pi}^{(1)}, \exists r \in \mathbb{R}, \exists c>0 / \forall \psi \in \mathcal{A}_{m}^{(1)}(\chi),|\lambda(\psi)| \leq c(m+1)^{r}
$$

and $\mathcal{J}$ the subset of $\mathcal{C}$ consisting of elements $\lambda$ satisfying the property

$$
\forall q \in \mathbb{R}, \exists c>0 / \forall \psi \in \mathcal{A}_{m}^{(1)}(\chi),|\lambda(\psi)| \leq c(m+1)^{q},
$$

we have the following proposition.

Proposition 1.6. If $T \in \mathcal{D}_{2 \pi}^{\prime(n)}$, we set $\langle T, u\rangle=\left(T, u\left(\psi^{\otimes n}\right)\right)_{\psi}$ for $u \in \mathcal{X}_{n}$ and $\psi \in \mathcal{A}^{(1)}$, then $T\left(\mathcal{X}^{(n)}\right) \subset \mathcal{C}$ and $T\left(\mathcal{N}^{(n)}\right) \subset \mathcal{J}$.

Obviously $\mathcal{C}$ is an algebra and $\mathcal{J}$ is an ideal of $\mathcal{C}$, then we have the definition

Definition 1.5. The algebra of generalized complex numbers is defined as $\overline{\mathcal{C}}=\mathcal{C} / \mathcal{J}$.

To each complex number $z$ we can assign the class in $\overline{\mathcal{C}}$ of the constant map $\lambda$ such that $\lambda(\psi)=z$ for all $\psi \in \mathcal{A}^{(1)}$. Thus we define a morphism between the algebras $\mathbb{C}$ and $\overline{\mathcal{C}}$ which is clearly a canonical embedding; we denote it by $\overline{\mathbf{i}}$. For any $n \in \mathbb{N}$ there also exist a canonical embedding of $\overline{\mathcal{C}}$ in $\mathcal{G}_{n}$ as follows. If $\Lambda$ denotes a generalized complex number with $\lambda$ as representative, then we assign to it the element of $\mathcal{G}_{n}$ which is the class of $\left[\psi^{\otimes n} \longmapsto \lambda(\psi)\right]$. Obviously this class does not depend on the chosen representative $\lambda$. Then we define a one-to-one morphism which is denoted by $\overline{\mathbf{j}}$.

Definition 1.6. An element $U$ of $\mathcal{G}_{n}$ will be called constant if there exists $\Lambda \in \overline{\mathcal{C}}$ such that $U=\overline{\mathbf{j}}(\Lambda)$.

Let $x \in \mathbb{R}^{n}$ and $U \in \mathcal{G}_{n}$. If $u$ and $v$ are two representatives of $U$ it is easy to see that $\left[\psi \longmapsto(u-v)\left(\psi^{\otimes n}\right)(x)\right]$ is an element of $\mathcal{J}$, thus we give the following definition:

Definition 1.7. Let $x \in \mathbb{R}^{n}$ and $U \in \mathcal{G}_{n}$. Then, the pointvalue of $U$ at $x$ is the generalized complex number $U(x)=\operatorname{cl}\left[\psi \longmapsto u\left(\psi^{\otimes n}\right)(x)\right]$. 
Clearly if $U$ is constant then $U$ takes contant pointvalues but it is shown that the converse is false. The notion of association in $\overline{\mathcal{C}}$ is provided by the following definition:

Definition 1.8. Two elements $\Lambda$ and $\Lambda^{\prime}$ in $\overline{\mathcal{C}}$ are said to be associated if they admit representatives $\lambda$ and $\lambda^{\prime}$ such that $\lim _{m \rightarrow \infty}\left[\lambda\left(\rho_{m}\right)-\lambda^{\prime}\left(\rho_{m}\right)\right]=0 \in \mathbb{C}$; we write $\Lambda \approx \Lambda^{\prime}$. We say that $\Lambda$ is associated with $z \in \mathbb{C}$ if $\Lambda \approx \overline{\mathbf{i}}(z)$, that is $\lim _{m \rightarrow \infty} \lambda\left(\rho_{m}\right)=z$.

Obviously the association in $\overline{\mathcal{C}}$ is an equivalence relation. For $\varphi \in \mathcal{A}^{(n)}$ let $o(\varphi)=\sup \left\{m \in \mathbb{N} \mid \varphi \in \mathcal{A}_{m}^{(n)}\right\}$. Then the pointvalue of $\left[\mathbf{j}\left(\delta_{n}\right)\right]$ at the point $x \neq 0$ is:

$$
\left[\mathbf{j}\left(\delta_{n}\right)\right](x)=\mathrm{cl}\left[\prod_{k=1}^{n} \frac{\sin (o(\psi)+1 / 2) x_{k}}{\sin \left(x_{k} / 2\right)}\right]_{\psi} .
$$

It may be seen that $\left[\mathbf{j}\left(\delta_{n}\right)\right](0)=\operatorname{cl}\left((2 m+1)^{n}\right)$. Subsequently we shall denote this last equality by $\left[\mathbf{j}\left(\delta_{n}\right)\right](0) \approx+\infty$.

Nonlinear operations in $\mathcal{G}_{n}$ and $\overline{\mathcal{C}}$. For $k \in \mathbb{N}$, let $F$ denote a complex valued function defined on $\mathbb{R}^{n} \times \mathbb{C}^{k}$ which is $2 \pi$-periodic with respect to the first variable. We suppose that $F$ is smooth on $\mathbb{R}^{n} \times \mathbb{R}^{2 k}$ when $\mathbb{C}$ is identified with $\mathbb{R}^{2}$ and $\mathbb{C}^{k}$ with $\mathbb{R}^{2 k}$, and satisfies the following condition:

$$
\begin{aligned}
& \forall(\alpha, \beta, \gamma) \in \mathbb{N}^{n} \times \mathbb{N}^{k} \times \mathbb{N}^{k}, \exists M>0, \exists \mu>0, \forall x \in \mathbb{R}^{n}, \\
& \left|(\partial / \partial x)^{\alpha}(\partial / \partial \xi)^{\beta}(\partial / \partial \eta)^{\gamma} F(x, \xi+i \eta)\right| \leq M(1+\|\xi+i \eta\|)^{\mu}
\end{aligned}
$$

where $(\xi, \eta) \in \mathbb{R}^{2 k}$ and $\xi+i \eta=\left(\xi_{1}+i \eta_{1}, \ldots, \xi_{k}+i \eta_{k}\right)$. If $u_{1}, \ldots, u_{k}$ are $k$ elements in $\mathcal{X}_{n}$, we set $F\left(u_{1}, \ldots, u_{k}\right)=\left[F\left(u_{1}(\varphi), \ldots, u_{k}(\varphi)\right)\right]_{\varphi}$, which is obviously an element of $\mathcal{X}_{n}$. We have:

Proposition 1.7. If $u_{1}, \ldots, u_{k}$ are $k$ elements in $\mathcal{X}^{(n)}$, then $F\left(u_{1}, \ldots, u_{k}\right)$ $\in \mathcal{X}^{(n)}$. Moreover if $v_{1}, \ldots, v_{k}$ are $k$ elements in $\mathcal{X}^{(n)}$ such that $u_{i}-v_{i} \in$ $\mathcal{N}^{(n)}$ for $i=1, \ldots, k$, then $F\left(u_{1}, \ldots, u_{k}\right)-F\left(v_{1}, \ldots, v_{k}\right) \in \mathcal{N}^{(n)}$.

Now, if $\left(U_{1}, \ldots, U_{k}\right) \in\left(\mathcal{G}_{n}\right)^{k}$, we may define $F\left(U_{1}, \ldots, U_{k}\right)=\operatorname{cl}\left[F\left(u_{1}, \ldots\right.\right.$, $\left.u_{k}\right)$ ] where $u_{1}, \ldots, u_{k}$ are arbitrary representatives of $U_{1}, \ldots, U_{k}$ respectively. In the same way, if $\left(\Lambda_{1}, \ldots, \Lambda_{k}\right) \in \overline{\mathcal{C}}^{k}$ we set $F\left(\Lambda_{1}, \ldots, \Lambda_{k}\right)=$ cl $\left[F\left(\lambda_{1}, \ldots, \lambda_{k}\right)\right]$ where $\lambda_{1}, \ldots, \lambda_{k}$ are representatives of $\Lambda_{1}, \ldots, \Lambda_{k}$ respectively. In this last case derivatives are not taken in account and no assumption of regularity on $F$ are needed. 


\section{The Goursat problem}

\subsection{The generalized Goursat problem.}

Let $F$ be a function defined on $\mathbb{R}^{2} \times \mathbb{C}$ which is smooth on $\mathbb{R}^{4}$ when one identifies $\mathbb{C}$ with $\mathbb{R}^{2}$. We suppose that $F$ satisfies the following conditions:

$\left(\mathbf{H}_{0}\right) F$ is an odd function, separately in the variables $x$ and $y$, and $2 \pi$-periodic with respect to these variables, that is

$$
\begin{aligned}
& \forall(x, y, z) \in \mathbb{R}^{2} \times \mathbb{C},\left\{\begin{array}{ll}
F(x+2 \pi, y, z) & =F(x, y+2 \pi, z)=F(x, y, z) \\
F(-x, y, z) & =F(x,-y, z)=-F(x, y, z)
\end{array} ;\right. \\
& \left(\mathbf{H}_{1}\right) k=\sup _{(x, y, z) \in \mathcal{I}^{2} \times \mathbb{C}}\left[\left|\frac{\partial F}{\partial z}(x, y, z)\right|+\left|\frac{\partial F}{\partial \bar{z}}(x, y, z)\right|\right]<+\infty
\end{aligned}
$$

$\left(\mathbf{H}_{2}\right) F$ and all its derivatives are slowly increasing at infinity uniformly with respect to $(x, y)$, that is

$$
\begin{aligned}
& \forall \alpha=\left(\alpha_{1}, \alpha_{2}, \alpha_{3}, \alpha_{4}\right) \in \mathbb{N}^{4}, \exists c>0, \exists \gamma>0 / \\
& \forall(x, y, z) \in \mathbb{R}^{2} \times \mathbb{C},\left|\frac{\partial^{|\alpha|} F}{\partial x^{\alpha_{1}} \partial y^{\alpha_{2}} \partial z^{\alpha_{3}} \partial \bar{z}^{\alpha_{4}}}(x, y, z)\right| \leq c(1+|z|)^{\gamma} .
\end{aligned}
$$

Let $V$ and $W$ be two elements of $\mathcal{G}_{1}$ such that

$\left(\mathbf{H}_{3}\right) V(0)=W(0)$.

$\left(\mathbf{H}_{4}\right) V$ and $W$ admit representatives with images consisting of even functions.

Then we want to find a solution in $\mathcal{G}_{2}$ to the following problem:

$$
\begin{cases}\frac{\partial^{2} U}{\partial x \partial y} & =F(., ., U) \\ U_{\mid\{y=0\}} & =V \\ U_{\mid\{x=0\}} & =W .\end{cases}
$$

Let us point out that because of $\left(\mathbf{H}_{3}\right), V$ and $W$ admit representatives $v$ and $w$ such that $v(\psi)(0)=w(\psi)(0)$ for any $\psi$ in $\mathcal{A}^{(1)}$. To see this let $v$ and $g$ be representatives of $V$ and $W$. If we define $\lambda$ in $\mathcal{X}^{(1)}$ by $\lambda(\psi)=$ $v(\psi)(0)-g(\psi)(0)$ then from $\left(\mathbf{H}_{3}\right)$ we have $\Lambda=\operatorname{cl}(\lambda)=0$ in $\mathcal{G}_{1}$. Now, let $w=g+\lambda$. Then $v(\psi)(0)=w(\psi)(0)$ for any $\psi$ in $\mathcal{A}^{(1)}$ and $W=\operatorname{cl}(w)$. Moreover since $\lambda(\psi)$ is even for any $\psi$ in $\mathcal{A}^{(1)}$, we may suppose that $v(\psi)$ and $w(\psi)$ are even for any $\psi$ in $\mathcal{A}^{(1)}$.

Subsequently $v$ and $w$ denote representatives of $V$ and $W$ satisfaying the conditions above, that is:

$$
\begin{aligned}
& \left(\mathbf{H}_{3}^{\prime}\right) \forall \psi \in \mathcal{A}^{(1)}, v(\psi)(0)=w(\psi)(0) ; \\
& \left(\mathbf{H}_{4}^{\prime}\right) \forall \psi \in \mathcal{A}^{(1)}, \forall t \in \mathbb{R}, \begin{cases}v(\psi)(-t) & =v(\psi)(t) \\
w(\psi)(-t) & =w(\psi)(t) .\end{cases}
\end{aligned}
$$


We set $h(t)=\sum_{n>0} t^{n} /(n !)^{2}$ and $\mathbf{1}(t)=1 ; \delta$ stands for the $2 \pi$-periodic Dirac measure on $\mathbb{R}$. In the sequel, to avoid confusions, $\delta_{x}$ (resp. $\delta_{y}$ ) means that $\delta$ acts on functions of the variable $x$ (resp. $y$ ).

\subsection{Statement of the main results.}

Theorem 2.1. Under conditions $\left(\mathbf{H}_{0}\right)-\left(\mathbf{H}_{4}\right)$, the Goursat problem $(\mathbf{I})$ admits a unique solution $U$ in $\mathcal{G}_{2}$. Moreover $U$ has a representative $u$ such that for any $\varphi$ in $\mathcal{A}^{(2)}, u(\varphi)$ is even with respect to each variable.

Let $F$ be defined by $F(x, y, z)=F_{1}(x) F_{2}(y) z$ where $F_{1}$ and $F_{2}$ are two odd functions belonging to $\mathcal{E}_{2 \pi}^{(1)}$. We denote by $\theta_{1}$ and $\theta_{2}$ the respective primitives of $F_{1}$ and $F_{2}$ taking the value 0 at the origine. Setting

$$
\nu_{1}(x, y, t)=\theta_{2}(y)\left(\theta_{1}(x)-\theta_{2}(t)\right) \quad \text { and } \quad \nu_{2}(x, y, t)=\theta_{1}(x)\left(\theta_{1}(y)-\theta_{2}(t)\right),
$$

we have the following proposition:

Proposition 2.2. Under the conditions $\left(\mathbf{H}_{3}^{\prime}\right),\left(\mathbf{H}_{4}^{\prime}\right)$, as well as $\left(\mathbf{H}_{3}\right),\left(\mathbf{H}_{4}\right)$, the Goursat problem $(\mathbf{I})$ admits a unique solution $U$ in $\mathcal{G}_{2}$, defined by

$$
\begin{aligned}
u\left(\psi^{\otimes 2}\right)(x, y) & =v(\psi)(0) h\left(\theta_{1}(x) \theta_{2}(y)\right)+\int_{0}^{x}[v(\psi)]^{\prime}(t) h\left(\nu_{1}(x, y, t)\right) d t \\
& +\int_{0}^{y}[w(\psi)]^{\prime}(t) h\left(\nu_{2}(x, y, t)\right) d t .
\end{aligned}
$$

Moreover for $V=\mathbf{j}(\delta)$ and $W=\mathbf{j}(\delta)$ the following weak equality holds

$$
U \approx \mathbf{j}(\delta \otimes \mathbf{1}+\mathbf{1} \otimes \delta)-\delta(0)\left[h \circ\left(\theta_{1} \otimes \theta_{2}\right)\right] .
$$

Further when $F=0$ one has that $U=\operatorname{cl}\left(\psi^{\otimes 2} \longmapsto \psi \otimes \mathbf{1}+\mathbf{1} \otimes \psi\right)-\mathbf{j}(\delta)(0)$.

Since $[\mathbf{j}(\delta)](0) \approx+\infty$, we see that if mes $\left\{(x, y) \in \mathcal{I}^{2} / h\left(\theta_{1}(x) \theta_{2}(y)\right)=0\right\}$ $\neq 0$, in particular for $F=0, U$ is not associated with a distribution, even though the data belong to $\mathbf{j}\left(\mathcal{D}_{2 \pi}^{\prime(1)}\right)$.

Now suppose that $F(x, y, z)=F_{1}(x) F_{2}(y) z+R(x, y, z)$ whith $F_{1}$ and $F_{2}$ as above, $R$ being a smooth function of the real variables when one identifies $\mathbb{C}$ with $\mathbb{R}^{2}$.

Proposition 2.3. Suppose that $V=\mathbf{j}\left(\delta_{x}\right), W=\mathbf{j}\left(\delta_{y}\right)$ and $R$ satisfies $\left(\mathbf{H}_{0}\right)-\left(\mathbf{H}_{2}\right)$, together with

$\left(\mathbf{H}_{5}\right) \quad \forall(x, y) \in \mathcal{I}^{2}, \lim _{|z| \rightarrow+\infty} R(x, y, z)=0$.

$\left(\mathbf{H}_{6}\right) \operatorname{mes}\left\{(x, y) \in \mathcal{I}^{2} / h\left(\theta_{1}(x) \theta_{2}(y)\right)=0\right\}=0$.

Then the following weak equality holds:

$$
U \approx \mathbf{j}(\delta \otimes \mathbf{1}+\mathbf{1} \otimes \delta)-\delta(0)\left[h \circ\left(\theta_{1} \otimes \theta_{2}\right)\right] .
$$


In this case, we see that the corresponding system behaves as the linear one.

Example 2.1. If $F(x, y, z)=\sin x \cdot \sin y \cdot\left(z+\exp \left(-z^{2}\right)\right)$. We have $F_{1}(x)=$ $\sin x, F_{2}(y)=\sin y$ and $R(x, y, z)=\sin x \cdot \sin y \cdot \exp \left(-z^{2}\right)$. The conditions $\left(\mathbf{H}_{0}\right)-\left(\mathbf{H}_{6}\right)$ are obviously fulfilled; moreover $\theta_{1}(x)=1-\cos x$ and $\theta_{2}(y)=$ $1-\cos y$, and we obtain

$$
U \approx \mathbf{j}(\delta \otimes \mathbf{1}+\mathbf{1} \otimes \delta)-\delta(0) h[(1-\cos x)(1-\cos y)] .
$$

\subsection{Proof of the results.}

At first we begin with the following lemma related to the case of smooth data.

Lemma 2.4. For each fixed $\psi$ in $\mathcal{E}_{2 \pi}^{(1)}$, the problem

$$
\left(\mathbf{I}^{\prime}\right) \quad \begin{cases}\frac{\partial^{2} u}{\partial x \partial y}(x, y) & =F(x, y, u(x, y)) \\ u(x, 0) & =v(\psi)(x) \\ u(0, y) & =w(\psi)(y)\end{cases}
$$

admits a unique solution in $\mathcal{E}_{2 \pi}^{(2)}$, which is an even function with respect to each variable.

Proof of Lemma 2.4. For sake of simplicity we shall omit $\psi$ in our notation.

Existence of a solution. Set $u_{0}(x, y)=v(x)-w(y)-v(0)$. Then $u_{0}$ is a smooth $2 \pi$-periodic function which is even with respect to each variable. We define a sequence $\left(u_{n}\right)_{n \geq 0}$ of functions on $\mathbb{R}^{2}$ by induction on $n$ as follows:

$$
u_{n}(x, y)=u_{0}(x, y)+\int_{0}^{x} \int_{0}^{y} F\left(\xi, \eta, u_{n-1}(\xi, \eta)\right) d \xi d \eta
$$

We shall show that for each $n, u_{n}$ is $2 \pi$-periodic and even with respect to each variable; we proceed by induction.

We know that these properties are true for $u_{0}$. Suppose they hold for $u_{n-1}$ for some $n \geq 1$. From $\left(\mathbf{H}_{0}\right)$ we deduce that $\int_{0}^{x} \int_{0}^{y} F\left(\xi, \eta, u_{n-1}(\xi, \eta)\right) d \xi d \eta$ is even with respect to each of the variables $x$ and $y$, whence $u_{n}$ has the same property. On the other hand we have

$$
\begin{aligned}
u_{n}(x+2 \pi, y)-u_{n}(x, y) & =\int_{x}^{x+2 \pi} \int_{0}^{y} F\left(\xi, \eta, u_{n-1}(\xi, \eta)\right) d \xi d \eta \\
& =\int_{0}^{y} \int_{x}^{x+2 \pi} F\left(\xi, \eta, u_{n-1}(\xi, \eta)\right) d \xi d \eta
\end{aligned}
$$


and from $\left(\mathbf{H}_{0}\right)$ it follows that for any fixed $\eta, F\left(\xi, \eta, u_{n-1}(\xi, \eta)\right)$ defines an odd and $2 \pi$-periodic function of $\xi$, whence

$$
\int_{x}^{x+2 \pi} F\left(\xi, \eta, u_{n-1}(\xi, \eta)\right) d \xi=\int_{-\pi}^{\pi} F\left(\xi, \eta, u_{n-1}(\xi, \eta)\right) d \xi=0 .
$$

Thus we have $u_{n}(x+2 \pi, y)=u_{n}(x, y)$ and in the same way we can prove that $u_{n}(x, y+2 \pi)=u_{n}(x, y)$, whence we derive the $2 \pi$-periodicity of $u_{n}$. Now if $\left(u_{n}\right)_{n \geq 0}$ is convergent then its limit will be $2 \pi$-periodic and even with respect to each variable.

Let $(x, y) \in \mathcal{I}^{2}$. For any $\left(\xi, \eta, z, z^{\prime}\right)$ in $\mathcal{I}^{2} \times \mathbb{C}^{2}$ we have

$$
\begin{aligned}
F(\xi, \eta, z)-F\left(\xi, \eta, z^{\prime}\right) & =\left(z-z^{\prime}\right) \int_{0}^{1} \frac{\partial F}{\partial z}\left(\xi, \eta, z^{\prime}+t\left(z-z^{\prime}\right)\right) d t \\
& +\left(\overline{z-z^{\prime}}\right) \int_{0}^{1} \frac{\partial F}{\partial \bar{z}}\left(\xi, \eta, z^{\prime}+t\left(z-z^{\prime}\right)\right) d t
\end{aligned}
$$

whence we obtain by using condition $\left(\mathbf{H}_{1}\right)$

$$
\left|F\left(\xi, \eta, u_{0}(\xi, \eta)\right)\right| \leq|F(\xi, \eta, 0)|+k\left\|u_{0}\right\|_{\infty} .
$$

Setting $\phi_{n}=u_{n}-u_{n-1}$ and $c=\|F(., ., 0)\|_{\infty}+k\left\|u_{0}\right\|_{\infty}$, it follows that

$$
\left|\phi_{1}(x, y)\right| \leq c|x y|
$$

and by induction we obtain for $n \geq 1$

$$
\begin{aligned}
\left|\phi_{n}(x, y)\right| & \leq c \frac{k^{n-1}|x y|^{n}}{(n !)^{2}} \\
\left\|\phi_{n}\right\|_{\infty} & \leq c \frac{k^{n-1} \pi^{2 n}}{(n !)^{2}} .
\end{aligned}
$$

This last inequality shows the uniform convergence of the series $\sum_{n \geq 1} \phi_{n}$ on $\mathcal{I}^{2}$ and then, on every compact of $\mathbb{R}^{2}$ from periodicity. It follows that the sequence $\left(u_{n}\right)_{n \geq 0}$ converges and its limit $u$ satisfies

$$
u(x, y)=u_{0}(x, y)+\int_{0}^{x} \int_{0}^{y} F(\xi, \eta, u(\xi, \eta)) d \xi d \eta
$$

Thus we derive that $u$ is a smooth function solving $\left(\mathbf{I}^{\prime}\right)$.

Uniqueness of the solution. We denote by $u_{1}$ and $u_{2}$ two solutions of $\left(\mathbf{I}^{\prime}\right)$ and we set $\phi=u_{1}-u_{2}$. Since $u_{1}$ and $u_{2}$ solve the integral equation (1), we have from the mean value theorem that $|\phi(x, y)| \leq k\left|\int_{0}^{x} \int_{0}^{y}\right| \phi(\xi, \eta)|d \xi d \eta|$. Hence for any $(x, y)$ in $\mathcal{I}^{2}$ it follows that:

$$
|\phi(x, y)| \leq k \pi\left|\int_{0}^{x} \sup _{\eta \in \mathcal{I}}\right| \phi(\xi, \eta)|d \xi| .
$$


Setting $g(x)=\sup _{\eta \in \mathcal{I}}|\phi(x, \eta)|$ for $x \in \mathcal{I}$, we have $g(x) \leq k \pi\left|\int_{0}^{x} g(\xi) d \xi\right|$. By applying Gronwall's lemma we obtain $g=0$, whence $\phi=0$.

Proof of Theorem 2.1. Let $u$ denote the element of $\mathcal{X}_{2}$ which associates to each $\psi^{\otimes 2}$ with $\psi$ in $\mathcal{E}_{2 \pi}^{(1)}$, the unique solution of $\left(\mathbf{I}^{\prime}\right)$. First we have to prove that $u$ is in $\mathcal{X}^{(2)}$. In all what follows, whenever $\psi$ belongs to $\mathcal{A}^{(1)}$, we set $\varphi=\psi^{\otimes 2}$. Inserting $\psi$ in the previous, we obtain

$$
c(\psi)=\|F(., ., 0)\|_{\infty}+k\left\|u_{0}(\varphi)\right\|_{\infty} \quad \text { and } \quad\left\|\phi_{n}(\varphi)\right\|_{\infty} \leq c(\psi) \frac{k^{n-1} \pi^{2 n}}{(n !)^{2}} .
$$

Observing that $u_{n}(\varphi)=u_{0}(\varphi)+\sum_{n \geq 1} \phi_{n}(\varphi)$ and setting $s=\sum_{n \geq 1} k^{n-1} \pi^{2 n} /$ $(n !)^{2}$, we find

$$
\|u(\varphi)\|_{\infty} \leq\left\|u_{0}(\varphi)\right\|_{\infty}+c(\psi) s .
$$

Since $v$ and $w$ are elements of $\mathcal{X}^{(1)},\left(\left\|u_{0}(\varphi)\right\|_{\infty}\right)_{\psi}$ and $(c(\psi))_{\psi}$ are in $\mathcal{C}$, as is $\left(\|u(\varphi)\|_{\infty}\right)_{\psi}$. Thus (2) gives the estimate of first order, that is $\alpha=0$ in the definition of $\mathcal{X}^{(2)}$. Differentiating (1) with respect to $x$ gives

$$
(\partial / \partial x) u(\varphi)(x, y)=(\partial / \partial x) u_{0}(\varphi)(x, y)+\int_{0}^{y} F(x, \eta, u(\varphi)(x, \eta)) d \eta .
$$

From $\left(\mathbf{H}_{2}\right)$ there exist $c_{1}>0$ and $\gamma_{1}>0$ such that for any $(x, y) \in \mathcal{I}^{2}$,

$$
|F(x, \eta, u(\varphi)(x, \eta))| \leq c_{1}\left(1+\|u(\varphi)\|_{\infty}\right)^{\gamma_{1}} .
$$

Combining (2) and (3), we obtain

$$
\|(\partial / \partial x) u(\varphi)\|_{\infty} \leq\left\|(\partial / \partial x) u_{0}(\varphi)\right\|_{\infty}+\pi c_{1}\left(1+\|u(\varphi)\|_{\infty}\right)^{\gamma_{1}}
$$

and in the same way we conclude that $\left(\|(\partial / \partial x) u(\varphi)\|_{\infty}\right)_{\psi}$ is an element of $\mathcal{C}$. Then by induction we infer, by using $\left(\mathbf{H}_{2}\right)$, that $\left(\left\|\partial^{a} u(\varphi)\right\|_{\infty}\right)_{\psi}$ is in $\mathcal{C}$ for any $\alpha$ in $\mathbb{N}^{2}$. That is, $u$ is an element of $\mathcal{X}^{(2)}$.

We now prove that $U=\operatorname{cl}(u)$ is a solution of $(\mathbf{I})$ in $\mathcal{G}_{2}$. For this let $\nu$ be an element of $\mathcal{N}^{(2)}$. Then we have for $\varphi$ in $\mathcal{A}^{(2)}$ :

$$
\begin{aligned}
& (\partial / \partial x)(\partial / \partial y)[(u+\nu)(\varphi)]-F(., .,(u+\nu)(\varphi))=(\partial / \partial x)(\partial / \partial y)[\nu(\varphi)] \\
& -\nu(\varphi) \int_{0}^{1}(\partial / \partial z) F(., .,(u+t \nu)(\varphi)) d t-\bar{\nu} \int_{0}^{1}(\partial / \partial \bar{z}) F(., .,(u+t \nu)(\varphi)) d t
\end{aligned}
$$

Because of $\left(\mathbf{H}_{2}\right), \varphi \mapsto \int_{0}^{1}(\partial / \partial z) F(., ., u(\varphi)+t \nu(\varphi)) d t$ is an element of $\mathcal{X}^{(2)}$ and the same holds by interchanging $(\partial / \partial z) F$ and $(\partial / \partial \bar{z}) F$. Since $\nu$, and hence $\bar{\nu}$, are in $\mathcal{N}^{(2)}$ it follows that $[(\partial / \partial x)(\partial / \partial y)(u+\nu)-(F ., ., u+\nu)]_{\varphi}$ belongs to $\mathcal{N}^{(2)}$ as well. This proves that any representative of $U$ solves the first line of $\left(\mathbf{I}^{\prime}\right)$ modulo $\mathcal{N}^{(2)}$. Since obviously the others are satisfied in the same manner by $u+\nu, U$ is a solution of the problem $(\mathbf{I})$. 
We now prove the uniqueness of $U$. Let $U_{1}$ denote a solution of $(\mathbf{I})$ with representative $u_{1}$. Setting $\phi=u_{1}-u$ we must prove that $\phi$ belongs to $\mathcal{N}^{(2)}$. Since $U_{1}$ is a solution of $(\mathbf{I})$, there exist $\nu$ in $\mathcal{N}^{(2)}, \zeta$ and $\mu$ in $\mathcal{N}^{(1)}$ such that for any $\psi$ in $\mathcal{A}^{(1)}$ and any $(x, y)$ in $\mathcal{I}^{2}$,

$$
\begin{cases}\frac{\partial^{2} u_{1}(\varphi)}{\partial x \partial y}(x, y) & =F\left(x, y, u_{1}(\varphi)(x, y)\right)+\nu(\varphi)(x, y) \\ u_{1}(\varphi)(x, 0) & =v(\psi)(x)+\zeta(\psi)(x) \\ u_{1}(\varphi)(0, y) & =w(\psi)(y)+\mu(\psi)(y) .\end{cases}
$$

It follows that

$$
\begin{aligned}
\phi(\varphi)(x, y) & =\int_{0}^{x} \int_{0}^{y}\left[F\left(\xi, \eta, u_{1}(\varphi)(\xi, \eta)\right)-F(\xi, \eta, u(\varphi)(\xi, \eta))\right] d \xi d \eta \\
& +Z(\varphi)(x, y)
\end{aligned}
$$

where $Z(\varphi)=\zeta(\psi) \otimes \mathbf{1}+\mathbf{1} \otimes \mu(\psi)-\zeta(\psi)(0)$. Obviously $Z$ is an element of $\mathcal{N}^{(2)}$. Writing

$$
\begin{aligned}
\phi(\varphi)(x, y) & =\int_{0}^{x} \int_{0}^{y} \phi(\varphi)(\xi, \eta)\left[\int_{0}^{1}(\partial / \partial z) F(\xi, \eta,(u+t \phi)(\varphi)(\xi, \eta)) d t\right] d \xi d \eta \\
& +\int_{0}^{x} \int_{0}^{y} \overline{\phi(\varphi)}(\xi, \eta)\left[\int_{0}^{1}(\partial / \partial \bar{z}) F(\xi, \eta,(u+t \phi)(\varphi)(\xi, \eta)) d t\right] d \xi d \eta \\
& +Z(\varphi)(x, y),
\end{aligned}
$$

we find $|\phi(\varphi)(x, y)| \leq k\left|\int_{0}^{x} \int_{0}^{y}\right| \phi(\varphi)(\xi, \eta)|d \xi d \eta|+\|Z(\varphi)\|_{\infty}$, and using Gronwall's lemma as in the end of the proof of Lemma 2.4, gives

$$
\|\phi(\varphi)\|_{\infty} \leq\|Z(\varphi)\|_{\infty} \exp \left(k \pi^{2}\right) .
$$

Since $Z$ is an element of $\mathcal{N}^{(2)}$, we have that $\left(\|\phi(\varphi)\|_{\infty}\right)_{\psi}$ is in $\mathcal{J}$, and (5) gives the estimate of order 0 . Now by using the condition $\left(\mathbf{H}_{2}\right)$, we can prove by induction from (4) and (5), that for any $\alpha$ in $\mathbb{N}^{2},\left(\left\|\partial^{\alpha} \phi(\varphi)\right\|_{\infty}\right)_{\psi}$ is an element of $\mathcal{J}$ and hence $\phi$ belongs to $\mathcal{N}^{(2)}$, that is $U_{1}=U$.

Proof of Proposition 2.2. Here we have $F(x, y, z)=F_{1}(x) f_{2}(y) z$. Obviously $F$ satisfies the conditions $\left(\mathbf{H}_{0}\right)-\left(\mathbf{H}_{2}\right)$ and system $(\mathbf{I})$ takes the following form:

$$
\begin{cases}\frac{\partial^{2} U}{\partial x \partial y} & =\left(F_{1} \otimes F_{2}\right) U \\ U_{\mid\{y=0\}} & =V \\ U_{\mid\{x=0\}} & =W\end{cases}
$$


Explicit forms of the solution. We know from Theorem 2.1 that (II) has a uniqe solution. It is easy to see that this is the one given in Proposition 2.2 , because the function $h$ satisfies

$$
t h^{\prime \prime}(t)+h^{\prime}(t)-h(t)=0, \quad h(0)=1 .
$$

Integrating by parts in the expression of $u\left(\psi^{\otimes 2}\right)$ given in Proposition 2.2, one obtains

$$
\begin{aligned}
u\left(\psi^{\otimes 2}\right)(x, y) & =v(\psi, x)+w(\psi, y)-v(\psi)(0) h\left(\theta_{1}(x) \theta_{2}(y)\right) \\
& +\theta_{2}(y) \int_{0}^{x}[v(\psi)](t) F_{1}(t) h^{\prime}\left(\nu_{1}(x, y, t)\right) d t \\
& +\theta_{1}(x) \int_{0}^{y}[w(\psi)](t) F_{2}(t) h^{\prime}\left(\nu_{2}(x, y, t)\right) d t
\end{aligned}
$$

Case where $V=\mathbf{j}\left(\delta_{x}\right)$ and $W=\mathbf{j}\left(\delta_{y}\right)$. Here we take $v(\psi)(x)=\rho_{o(\psi)}(x)$ and $w(\psi)(y)=\rho_{o(\psi)}(y)$. Obviously $V$ and $W$ satisfy $\left(\mathbf{H}_{3}\right)$ and $\left(\mathbf{H}_{4}\right)$, hence this case is compatible with our assumptions.

We only study the first integral of (6), because the second one can be studied in a similar way. Since $F_{1}$ is an odd function, there exists a rapidly decreasing sequence $\left(a_{p}\right)_{p \geq 1}$ such that $F_{1}(t)=\sum_{p \geq 1} a_{p} \sin p t$. We set $\psi=$ $\rho_{m}$ and write

$$
\rho_{m}(t) \sin p t=\sin (m+1 / 2) t[\sin p t / \sin (t / 2)] .
$$

Since $|\sin p t / \sin (t / 2)| \leq 2 p$, one thus obtains

$$
\left|\rho_{m}(t) \sin p t\right| \leq 2 p .
$$

Therefore, setting $r=\sup \left\{\left|h^{\prime}\left(\nu_{1}(x, y, t)\right)\right| ;(x, y, t) \in \mathcal{I}^{3}\right\}$, it follows that

$$
\left|\rho_{m}(t) a_{p} \sin p t\right|\left|h^{\prime}\left(\nu_{1}(x, y, t)\right)\right| \leq 2 \operatorname{rrp}\left|a_{p}\right| .
$$

Inequality (8) shows that the series $\sum_{p \geq 1} h^{\prime}\left(\nu_{1}(x, y, t)\right) \rho_{m}(t) a_{p} \sin p t$ converges uniformly with respect to $t$. Thus we get

$$
\int_{0}^{x} \rho_{m}(t) F_{1}(t) h^{\prime}\left(\nu_{1}(x, y, t)\right) d t=\sum_{p=1}^{+\infty} a_{p} \int_{0}^{x} \rho_{m}(t) h^{\prime}\left(\nu_{1}(x, y, t)\right) \sin p t d t .
$$

On the other hand, (7) gives

$$
\left|\int_{0}^{x} \rho_{m}(t) h^{\prime}\left(\nu_{1}(x, y, t)\right) \sin p t d t\right| \leq 2 p r|x|
$$


which shows that the last series converges uniformly with respect to $p$ and then

$$
\begin{aligned}
& \lim _{m \rightarrow+\infty} \int_{0}^{x} \rho_{m}(t) F_{1}(t) h^{\prime}\left(\nu_{1}(x, y, t)\right) d t \\
& =\sum_{p=1}^{+\infty} a_{p}\left(\lim _{m \rightarrow+\infty} \int_{0}^{x} \rho_{m}(t) h^{\prime}\left(\nu_{1}(x, y, t)\right) \sin p t d t\right) .
\end{aligned}
$$

Now, from the Riemann-Lebesgue theorem it follows that

$$
\lim _{m \rightarrow+\infty} \int_{0}^{x} \rho_{m}(t) h^{\prime}\left(\nu_{1}(x, y, t)\right) \sin p t d t=0,
$$

and since

$$
\left|\theta_{2}(y) \int_{0}^{x}[v(\psi)](t) F_{1}(t) h^{\prime}\left(\nu_{1}(x, y, t)\right) d t\right| \leq 2 r\left|x \theta_{2}(y)\right| \sum_{p=1}^{+\infty} p\left|a_{p}\right|,
$$

Lebesgue's bounded convergence theorem gives

$$
\lim _{m \rightarrow+\infty} \theta_{2}(y) \int_{0}^{x}[v(\psi)](t) F_{1}(t) h^{\prime}\left(\nu_{1}(x, y, t)\right) d t=0 \quad \text { in } \quad \mathcal{D}_{2 \pi}^{\prime(2)} .
$$

We derive from this last equality and (6) that

$$
U+\delta(0)\left[h \circ\left(\theta_{1} \otimes \theta_{2}\right)\right] \approx \delta \otimes \mathbf{1}+\mathbf{1} \otimes \delta .
$$

Since for any element $T$ of $\mathcal{D}_{2 \pi}^{\prime(2)}$ the relation $\mathbf{j}(T) \approx T$ holds, we finally obtain

$$
U \approx \mathbf{j}(\delta \otimes \mathbf{1}+\mathbf{1} \otimes \delta)-\delta(0)\left[h \circ\left(\theta_{1} \otimes \theta_{2}\right)\right] .
$$

When $F=0$, we deduce directly from (II) that the solution is represented by

$$
u\left(\psi^{\otimes 2}\right)(x, y)=v(\psi, x)+w(\psi, y)-v(\psi)(0)
$$

and then

$$
U=\operatorname{cl}(\psi \longmapsto \psi \otimes \mathbf{1}+\mathbf{1} \otimes \psi)-\delta(0) .
$$

Proof of Proposition 2.3. We are concerned by the following system

$$
\left\{\begin{array}{l}
\frac{\partial^{2} U}{\partial x \partial y}=\left(F_{1} \otimes F_{2}\right) U+R(., ., U) \\
U_{\mid\{y=0\}}=V \\
U_{\mid\{x=0\}}=W .
\end{array}\right.
$$

Let $U_{1}$ denote the solution of (III) and $u_{1}$ the representative of $U_{1}$ such that for any $\psi$ in $\mathcal{A}^{(1)}, u_{1}\left(\psi^{\otimes 2}\right)$ is the solution of the associated system with 
$v(\psi)$ and $w(\psi)$ as initial data. If we set $\omega=u_{1}-u$ and $\psi^{\otimes}=\varphi$, then for any $\psi$ we have

$$
\begin{cases}\frac{\partial^{2} \omega(\varphi)}{\partial x \partial y} & =\left(F_{1} \otimes F_{2}\right)[\omega(\varphi)]+R(., ., \omega(\varphi)) \\ {[\omega(\varphi)]_{\mid\{y=0\}}} & =0 \\ {[\omega(\varphi)]_{\mid\{x=0\}}} & =0 .\end{cases}
$$

From here it follows as in (1) that

$$
\begin{aligned}
\omega(\varphi)(x, y) & =\int_{0}^{x} \int_{0}^{y} F_{1}(\xi) F_{2}(\eta) \omega(\varphi)(\xi, \eta) d \xi d \eta \\
& +\int_{0}^{x} \int_{0}^{y} R(\xi, \eta, \omega(\varphi)(\xi, \eta)) d \xi d \eta .
\end{aligned}
$$

Setting $g(\varphi, x)=\sup _{\eta \in \mathcal{I}}|\omega(\varphi)(x, \eta)|$ for $x \in \mathcal{I}$, we obtain

$$
g(\varphi, x) \leq \pi\left\|F_{1}\right\|_{\infty}\left\|F_{2}\right\|_{\infty}\left|\int_{0}^{x} g(\varphi, \xi) d \xi\right|+\pi^{2}\|R\|_{\infty} .
$$

Now, from Gronwall's lemma follows

$$
\|\omega(\varphi)\|_{\infty} \leq \pi^{2}\|R\|_{\infty} \exp \left(\pi^{2}\left\|F_{1}\right\|_{\infty}\left\|F_{2}\right\|_{\infty}\right) .
$$

From (6), there exists $\kappa \in \mathcal{X}^{(2)}$ such that $\lim _{m \rightarrow+\infty} \kappa\left(\rho_{m}^{\otimes 2}\right)(x, y)=0$ for any $(x, y)$ in $\mathcal{I}^{2}$, and

$$
u\left(\rho_{m}^{\otimes 2}\right)+\rho_{m}^{\otimes 2}(0)\left[h \circ\left(\theta_{1} \otimes \theta_{2}\right)\right]=\rho_{m} \otimes \mathbf{1}+\mathbf{1} \otimes \rho_{m}+\kappa\left(\rho_{m}^{\otimes 2}\right) .
$$

Let $\mathcal{H}$ denote the set $\left\{(x, y) \in \mathcal{I}^{2} \mid x y \neq 0, h\left(\theta_{1}(x) \theta_{2}(y)\right) \neq 0\right\}$. From $\left(\mathbf{H}_{4}\right)$, we derive that $\operatorname{mes}\left(\mathcal{I}^{2} \backslash \mathcal{H}\right)=0$. On the other hand, if $(x, y)$ belongs to $\mathcal{H}$, we have

$$
\left|\rho_{m}(x)\right| \leq 1 /|\sin (x / 2)| \quad \text { and } \quad\left|\rho_{m}(y)\right| \leq 1 /|\sin (y / 2)|
$$

from which we find

$$
\begin{aligned}
& \left|u\left(\rho_{m}^{\otimes 2}\right)(x, y)+\rho_{m}^{\otimes 2}(0) h\left(\theta_{1}(x) \theta_{2}(y)\right)\right| \\
& \leq 1 /|\sin (x / 2)|+1 /|\sin (y / 2)|+\left|\kappa\left(\rho_{m}^{\otimes 2}\right)(x, y)\right| .
\end{aligned}
$$

Since $\lim _{m \rightarrow+\infty} \rho_{m}(0)=+\infty, \lim _{m \rightarrow+\infty} \kappa\left(\rho_{m}^{\otimes 2}\right)(x, y)=0$ and $h\left(\theta_{1}(x) \theta_{2}(y)\right)$ $\neq 0$, we have $\lim _{m \rightarrow+\infty}\left|u\left(\rho_{m}^{\otimes 2}\right)(x, y)\right|=+\infty$. That is $\lim _{m \rightarrow+\infty}\left|u\left(\rho_{m}^{\otimes 2}\right)\right|=$ $+\infty$ almost everywhere in $\mathcal{I}^{2}$. Then from $(9), \lim _{m \rightarrow+\infty}\left|u_{1}\left(\rho_{m}^{\otimes 2}\right)(x, y)\right|=$ $+\infty$ a.e. in $\mathcal{I}^{2}$, and from $\left(\mathbf{H}_{3}\right)$ it follows that $\lim _{m \rightarrow+\infty} R\left(., ., u_{1}\left(\rho_{m}^{\otimes 2}\right)\right)=0$ a.e. in $\mathcal{I}^{2}$. Now, since $R$ is bounded, Lebesgue's bounded convergence theorem gives $\lim _{m \rightarrow+\infty} \iint_{\mathcal{I}^{2}}\left|R\left(\xi, \eta, \omega\left(\rho_{m}^{\otimes 2}\right)(\xi, \eta)\right)\right| d \xi d \eta=0$. On the other hand, it is obvious that we can interchange $\iint_{\mathcal{I}^{2}}\left|R\left(\xi, \eta, \omega\left(\rho_{m}^{\otimes 2}\right)(\xi, \eta)\right)\right| d \xi d \eta$ and $\pi^{2}\|R\|_{\infty}$ in (9). It follows that $\lim _{m \rightarrow+\infty} u_{1}\left(\rho_{m}^{\otimes 2}\right)=0$ in the subspace of continuous functions of $\mathcal{F}_{2 \pi}^{(2)}$, hence $\lim _{m \rightarrow+\infty} u_{1}\left(\rho_{m}^{\otimes 2}\right)=0$ in $\mathcal{D}_{2 \pi}^{\prime(2)}$. We 
have shown that $U$ and $U_{1}$ are associated, the desired result follows immediately.

\title{
References
}

[1] Biagioni, H. A., A Nonlinear Theory of Generalized Functions, Lectures Notes in Math. 1421 (1990), Springer-Verlag, Berlin-Heildelberg, New-York.

[2] Chou, C.-C., Séries de Fourier et Théorie des Distributions, Editions Scientifiques, Beijing, 1983.

[3] Colombeau, J. F., Elementary Introduction to New Generalized Functions, NorthHolland Math. Stud. 113 (1985), North-Holland, Amsterdam.

[4] Colombeau, J. F., New Generalized Functions and Multiplication of Distributions, North-Holland Math. Stud. 84 (1984), North-Holland, Amsterdam.

[5] Colombeau, J. F., Multiplication of distributions, Bull. Amer. Math. Soc. 23 (1990), 251-268.

[6] Egorov, Yu. V., A contribution to the theory of generalized functions, Russian Math. Survey 45(5) (1990), 1-49.

[7] Garabedian, P. R., Partial Differential Equations, John Wiley \& Sons, Inc., New York-London-Sydney, 1964.

[8] Goursat, E., Cours d'Analyse Mathématique, tome III, Gauthier-Villars, Montrouge, 1956.

[9] Oberguggenberger, M., Multiplication of Distributions and Applications to Partial Differential Equations, Pitman Res. Notes Math. Ser. 259 (1992), Longman Sci. Tech., Harlow.

[10] Oberguggenberger, M., Nonlinear theory of generalized functions, in "Advances in Analysis, Probability and Mathematical Physics - Contribution from Non Standard Analysis", Kluwer, Dordrecht, 1994.

[11] Rosinger, E., Generalized Solutions of Nonlinear Partial Differentiable Equations, North-Holland Math. Stud. 146 (1987), North-Holland, Amsterdam.

[12] Schwartz, L., Sur l'impossibilité de la multiplication des distributions, C. R. Acad. Sci. Paris Sér. I Math. 239 (1954), 847-848.

[13] V. Valmorin, Fonctions généralisées périodiques et problème de Goursat, C. R. Acad. Sci. Paris Sér. I Math. 320 (1995), 537-540.

[14] V. Valmorin, A new algebra of periodic generalized functions, Zeitschrift für Analysis und ihre Anwendungen, 15(1) (1996), 57-74.

[15] V. Valmorin, Fonctions Généralisées Périodiques et Applications, Dissertationes Math. 361 (1997), Polish Acad. Sci., Warsaw.

\author{
V. VALMORIN \\ Université des Antilles et de la Guyane \\ DÉPARTEMENT MATH-INFO \\ Campus de Fouillole: \\ 97159 Pointe À Pitre Cedex \\ French West Indies \\ VINCENT.VALMORIN@UNIV-AG.FR
}

